\title{
Magnetic activity and evolution of low-mass young stars
}

\author{
Thierry Montmerle \\ Service d'Astrophysique, Centre d'Etudes Nucléaires de Saclay \\ 91191 Gif-sur-Yvette, France
}

\section{X-rays from star-forming regions}

\subsection{Main observational results}

X-rays turned out rather unexpectedly to be of fundamental importance in studying, and especially in discovering, pre-main sequence stars. The bulk of the data we now have comes from observations using the Einstein satellite, operating in the imaging mode between $\sim 0.4$ to $\sim 4 \mathrm{keV}$ (for reviews, see, e.g., Feigelson 1984, Feigelson, Giampapa, and Vrba 1990); because many of the detected sources turned out to suffer a relatively large absorption ( $\mathrm{A}_{\mathrm{y}}$ up to several magnitudes, see below), EXOSAT, sensitive to softer X rays, unfortunately proved to be of little use.

Most of the observed regions were nearby dark clouds, well known to undergo active star formation and containing many T Tauri stars (low-mass pre-main sequence stars): $\rho$ Ophiuchi (Montmerle et al.1983), Taurus-Auriga (Feigelson et al. 1987, Walter et al. 1988), Chameleon (Feigelson and Kriss 1988), all at distances $~ 160$ pc, and Orion (Ku, RighiniCohen, and Simon 1982, Caillault and Zoonematkermani 1987) at $\sim 450 \mathrm{pc}$. Note that $\rho$ Oph and Orion in addition contain stars of earlier spectral types, the earliest being B3 and 07, respectively, themselves X-ray emitters. Note also the late publication date of some papers, which reflects the very contribution of the X-rays: many of the stellar X-ray sources discovered were previously unknown, and it took often several years to establish (spectroscopically) their pre-main sequence nature.

All these observations revealed dozens of new sources, and in many cases, the Xrays revealed more PMS stars (by a factor $f>1$ ) than were discovered by the usual criteria based on the presence of strong emission lines, notably $\mathrm{H} \alpha$. Although a definite figure cannot be given for lack of completeness in the various surveys, some authors (e.g., Walter et al. 1988) argue that $f \lesssim 10$.

\subsection{Emission mechanism}

When a good X-ray spectrum of a PMS star is available (which is not always the case at the level of sensitivity of Einstein because in general too few counts are recorded), it is consistent with bremsstrahlung emission from a hot $(\mathrm{kT} \sim 1 \mathrm{keV})$ plasma, significantly absorbed along the line of sight $\left(\mathrm{N}_{\mathrm{H}} \leqslant\right.$ a few $10^{21} \mathrm{~cm}^{-2}$, or $\left.\mathrm{A}_{\mathrm{V}} \lesssim 10\right)$. The typical luminosities are in the range $\left\langle\mathrm{L}_{\mathrm{x}}>\sim 10^{31-32}\right.$ erg.s $\mathrm{s}^{-1}$.

But more significant results can be gained from the study of time variability. Much evidence has accumulated in favor of the existence of large X-ray flares (e.g., Feigelson and DeCampli 1981, Walter and Kuhi 1984, Montmerle et al. 1983, 1984), which in fact dominate the X-ray emission. Within the still modest precision of the data, these flares are solar-like, but enhanced by factors up to $10^{6}$ with respect to the Sun, also enhanced with respect to flares on dMe "flare stars" (see discussion in Montmerle et al. 1983), and quite similar to flares observed on RS CVn close binary systems (e.g., Mutel et al. 1987). In addition to the 
solar analogy, many factors, including the presence of large spots and a correlation with rotation based on them (Montmerle 1987, Bouvier and Bertout 1989), argue in favor of a magnetic origin for this X-ray activity. However, strong flaring activity takes place $\sim 5 \%$ of the time on average (Gahm 1989).

\subsection{Implications on the stellar environment}

Interpreting the X-rays from PMS stars in terms of a magnetically confined plasma, the emission measure yields the typical size $\mathrm{H}_{\mathrm{X}}$ of the magnetic loops: one finds $\mathrm{H}_{\mathrm{X}} \leqslant 2-3 \mathrm{R}_{*}$. In turn, one deduces a typical value for the surface magnetic field: $B_{*, X} \sim$ a few $100 \mathrm{G}$. Whereas the size of the loops is large when compared to the Sun, $B_{*, x}$ is on the contrary quite representative of the solar active regions.

The width of the $H \alpha$ line associated with chromospheric activity is small, a few $\AA$ at most, and this is indeed the case for most of the X-ray discovered PMS stars. But in this context, it is surprising that the width of the $\mathrm{H} \alpha$ line turns out to be much larger (up to a few $100 \AA$ ) for all the previously known PMS stars like T Tauri stars, for a comparable X-ray flux (e.g., Bouvier 1987, shown in Montmerle 1987). Such an $\mathrm{H} \alpha$ "excess" goes along with other excesses (over a normal, late-type photosphere), in the UV and the near-IR, observed in the continuum spectra of these stars. These excesses are clearly "non-solar", and point to the existence, in the vicinity of the star, of circumstellar material, made up of hot $\left(\sim 10^{4} \mathrm{~K}\right)$ ionized gas, as well as warm $(\lesssim 1000 \mathrm{~K})$ dust.

Current models (Kenyon and Hartmann 1987, Bertout, Bouvier, and Basri 1988, Bertout 1989) attribute these excesses to the presence of an accretion disk, of size $\lesssim 1000 \mathrm{R}_{*}$. This disk is likely in keplerian rotation; in the immediate vicinity of the star, the corresponding orbital velocity is typically $\sim 250 \mathrm{~km} \cdot \mathrm{s}^{-1}$, whereas the stellar equatorial velocity is $\sim 20 \mathrm{~km} . \mathrm{s}^{-1}$. This introduces a new, important component in the circumstellar environment, in the form of a hot boundary layer. It is this layer which is thought, in these models, to be responsible for the strong emission lines, including $\mathrm{H} \alpha$. The corresponding stars are now called "classical" T Tauri stars ("CTTS").

By contrast, the X-ray discovered PMS stars, which do not show these excesses, were named "naked" T Tauri stars (Walter 1987), but the term "weak-line" T Tauri star ("WTTS") is now to be preferred, since it does not imply that the absence of IR and/or UV excess means an absence of circumstellar material (see §3.1).

\section{Radio observations in the cm range}

\subsection{Rationale}

Given the links between X-rays and magnetic activity, it is natural to look for non-thermal radio emission from the flare-associated electrons in the magnetic loops. This emission is observed on the Sun, and the above evidence for solar-type activity reinforces the potential interest of radio observations. But another type of radio emission is also expected: thermal free-free emission associated with the hot circumstellar environment.

Taking advantage of the increased sensitivity and the imaging capability in the $\mathrm{cm}$ $(\mathrm{GHz})$ range offered by the Very Large Array in New Mexico, a number of workers have done radio observations following two approaches. In the first approach, known objects, selected according to a variety of criteria, are the targets of pointed observations: CTTS (e.g. Bieging, Cohen, and Schwartz 1984), sources of molecular outflows (related PMS objects, see Snell and Bally 1986; Lada 1985). In the second approach, a whole region is subject to an 
unbiased survey, without any prior selection of targets.

The first approach gives evidence that a (small) number of CTTS or molecular flow sources have a detectable radio flux, of thermal origin, and confirms the existence of ionized stellar winds, already suspected to be present on the basis of some $\mathrm{H} \alpha$ and $\mathrm{NaD}$ emission line profiles (e.g., Mundt 1984). The observed mass-loss rates are typically $\dot{\mathrm{M}} \sim 10^{-8} \mathrm{M}_{\odot} \mathrm{yr}^{-1}$, velocities $v_{W} \sim 200-300 \mathrm{~km} . \mathrm{s}^{-1}$, and the size of the emitting region is $R \sim 1000 R_{*}$ (e.g., Panagia and Felli 1975).

The second approach makes no assumption about the nature of the possible radio emitters, and has revealed the existence of hitherto unknown objects, in p Oph (André, Montmerle, and Feigelson 1987), CrA (Brown 1987), and Orion (Trapezium region, Garay, Moran, and Reid 1987, Churchwell et al. 1987). Several stellar radio sources had been previously found in X-rays, but most were discovered deeply embedded in the clouds, so that even the X-rays, if present, would be absorbed. Here again, the study of time variability proved crucial in understanding the nature of the radio emission: strong variability was noticed as early as 1985, with the discovery of the first radio flare in a PMS star (Feigelson and Montmerle 1985), and was later established to be rather frequent among PMS radio emitters. The typical timescale for variability is unknown, but variability of factors of at least 2 have been found in a few hours, and up to 10 between observations separated by a few months (e.g., Cohen and Bieging 1986). Such timescales can be explained only in terms of a nonthermal emission mechanism, because the interpretation in terms of an ionized wind implies timescales $\gtrsim$ years. Note that the commonly used criteria based on the spectral index $\alpha$ (where $S_{v} \propto v^{\alpha}$ ), namely that if $\alpha>0$ the emission is thermal and if $\alpha<0$ the emission is nonthermal, cannot reliably indicate the nature of the emission mechanism, but simply indicates the opacity (resp. optically thick, and optically thin). (For details, see André 1987.)

\subsection{Non-thermal radio emission}

As mentioned above, there are indications that nonthermal radio emitters are not unusual among the whole population of PMS radio emitters. But the only systematic search to date has been undertaken in the $\rho$ Oph cloud, over a $\sim 2^{\circ} \times 2^{\circ}$ area, following the Einstein results (André, Montmerle, and Feigelson 1987, Stine et al. 1988). Forgetting about spectra, the nonthermal nature of a source can be established in three ways:

口 large-amplitude variability over short timescales ( $\Sigma$ years, see above);

a polarization;

u unresolved size (i.e., $<0.1^{n}$ at $15 \mathrm{GHz}$ for the VLA in A configuration at the distance of the nearest dark clouds).

As mentioned above, variability has been established in many cases, and, in $\rho 0 \mathrm{ph}$, appears to be widespread among the 9 sources found above 1-2 $\mathrm{mJy}$ (Stine et al. 1988). Also, none of these sources is resolved, as shown by a visibility analysis (see André 1987). More remarkably, in one instance, and for the first time, polarization (at a level of $\sim 7 \%$, and seen repeatedly) has been found in S1, a very young ( $\approx 2000 \mathrm{yrs}) \mathrm{B} 3$ star in $\rho$ Oph.

In all these examples, the emission mechanism is likely to be gyrosynchroton radiation from $\sim \mathrm{MeV}$ electrons spiraling in fields $\sim 1 \mathrm{G}$; at least, quantitative models exist for a few well-documented cases (DoAr21, see André 1987; S1, André et al. 1988), using nonhomogeneous emission models featuring dipolar magnetic loops, and developed for the 
Sun (e.g., Klein and Chiuderi-Drago 1987). The magnetic field at the stellar surface is in general $\mathrm{B}_{*, \mathrm{r}} \sim$ a few $100 \mathrm{G}$, consistent with the values $\mathrm{B}_{*, \mathbf{X}}$ deduced from the X-rays; in the case of $S 1$, however, a higher value is required $(\approx 10 \mathrm{kG})$, about the same as prevails in the well-known magnetic B stars.

All these stars share a remarkable feature: interpreting the radio emission in terms of the gyrosynchrotron mechanism, implies that they must possess extended magnetic structures, the size of which $\mathrm{H}_{\mathrm{r}}$ may extend up to 10 stellar radii (see Fig. 4 of Montmerle and André 1988), which is significantly larger than the size $\mathrm{H}_{\mathrm{x}}$ deduced from the X-rays. They therefore make up a new population of PMS stars, seen in several dark clouds but best known in $\rho$ Oph, and comprising to date about 15 members.

\section{Implications on evolution}

\subsection{Young stars and circumstellar material}

Contrary to the X-rays, which are spread between CTTS and WTTS (even though X-ray selected WTTS largely outnumber CTTS, as discussed in $\S 1.1$ ), there is in the radio range a clear distinction between emitters: the thermal mechanism concerns only CTTS and similar objects, and the nonthermal mechanism concerns only WTTS and related objects. There is also another strong difference between the X-rays and the radio: whereas the Xrays come from stars spread over a large area in the vicinity of a dark cloud, the nonthermal radio emitters (equivalently, those which have large magnetic structures) are found to be embedded in the clouds. The case of $\rho$ Oph is particularly striking: the 9 sources are strongly concentrated near the cloud core, whereas a large area $\left(\sim 2^{\circ} \times 2^{\circ}\right)$ has been searched with a good sensitivity $(\sim 2 \mathrm{mJy})$.

An immediate conclusion is that these objects must be very young, although, strictly speaking, no age can be attributed to them because most are not seen in the optical and hence cannot be placed on an HR diagram. But they are visible in the near-IR (e.g., Wilking, Lada, and Young 1989), and show no excess in this range. According to the IR classification (Lada 1988), they are "Class III" objects. So we are faced with a problem: how is it that very young stars, still embedded in cloud cores, apparently do not show evidence for circumstellar material?

A related problem exists when considering optically visible CTTS and WTTS as a whole. Indeed, when put on an HR diagram, these two classes of PMS stars appear mixed (e.g., Walter et al 1988, Edwards et al. 1987), whereas, according to "standard" evolutionary models (e.g., Adams, Lada, and Shu 1987), CTTS, being surrounded by accretion disks, should be younger, and WTTS, deprived of such disks, should represent a more advanced evolutionary stage.

\subsection{Cold circumstellar material and magnetic fields}

The probable answer to this problem lies in the fact that the "standard" scenario of early stellar evolution (Lada 1988, Adams et al. 1987) is based on IR data, from the near-IR $(\approx 2 \mu \mathrm{m})$, which traces warm material $(\approx 1500 \mathrm{~K})$, to the far-IR ( $\approx 100 \mu \mathrm{m}$, IRAS data), which traces colder material (few $100 \mathrm{~K}$ ). In current disk models (see above, $\S 1.3$ ), the temperature decreases outwards with radius according to a power law (index $-3 / 4$ in the case of a keplerian spatially thin viscous disk): the warm regions seen in the IR are comparatively close to the star $(\leqslant 1 \mathrm{AU})$, and the boundary layer exists only if there is a physical contact between the disk and the star. Outer $(\approx 10 \mathrm{AU})$, cold $(\ll 100 \mathrm{~K})$ material is 
visible only in the mm range, and recent work in this band has given new insight into the presence of circumstellar matter around young stars, and into the possible role of magnetic fields (Montmerle and André 1989).

Using the 30-m dish of the French-German IRAM telescope located on Pico Veleta near Granada (Spain), equipped with the sensitive MPIfR $1.3 \mathrm{~mm}$ bolometer, Beckwith et al. (1990) and André et al. (1990) have shown that about half of PMS stars, irrespective of their optical or IR classification, are strong emitters in the $\mathrm{mm}$ range, hence are surrounded by cold dust. More precisely, this is true for CTTS and WTTS (Beckwith et al. 1990), as well as for embedded IR sources in $\rho$ Oph, having strong IR excess to no excess (André et al. 1990). Combining the IR data with the mm data shows that a variety of circumstellar material (presumably disks) may exist around similar stars: mm emission without IR excess indicates the presence of "hollow", cold disks, whereas IR excess without $\mathrm{mm}$ emission shows the existence of "compact", warm disks; "normal", i.e., extended disks, are also present (see Fig. 1 of André et al. 1990).

Put in an evolutionary perpective, it therefore appears qualitatively that stars of (roughly) the same mass and age may be surrounded by mophologically different disks. The reason for this difference can be linked with the question of disk stability on timescales $10^{5}-10^{7} \mathrm{yrs}$, and magnetic fields may there play a crucial role (Montmerle and André 1989). For instance, Tagger et al. (1990) have recently shown that spatially thin accretion disks threaded by vertical magnetic field lines (as is the case for dipolar fields) are subject to efficient dynamical instabilities. Some of these instabilities may cause the inner parts of the disk, out to the corotation radius, to fall quickly on the star, resulting in the formation of an inner "hole". Much work remains to be done, however, before this can be quantitatively proven.

\section{Concluding remarks}

The environment of young stellar objects is complex, and involves a variety of interactions with them, on very different length scales: boundary layers $\left(r \gtrsim R_{*}\right)$, magnetic activity and magnetic fields $\left(r \lesssim 1-10 \mathrm{R}_{*}\right)$, disks $\left(r \lesssim 1000 \mathrm{R}_{*}\right)$, ionized winds $\left(\mathrm{r} \gtrsim 1000 \mathrm{R}_{*}\right)$ and molecular outflows $\left(\mathrm{r} \leqslant 10^{6} \mathrm{R}_{*}\right)$.

- X-rays and cm radio observations give crucial information on magnetic fields and on the existence of ionized circumstellar material; the UV range appears to be connected mainly with the existence of boundary layers.

a IR and $\mathrm{mm}$ radio observations give crucial information on the nature and extent of the outer, colder material; this material, presumably in the form of circumstellar disks, may be unstable on a wide range of timescales, from $\sim 10^{5}$ to $\sim 10^{7}$ years, plausibly owing to the presence of magnetic fields.

D One probably has to put together the information contained in all these energy domains, and the existence of magnetic fields, to better understand the mechanism(s) of star formation.

\section{References}

Adams F.C., Lada C.J., Shu F.H. 1987, Ap.J.312, 788.

André P., Montmerle T., Feigelson E.D. 1987, Astr.J.93, 1182.

André P., Montmerle T., Steppe H., Feigelson 1990, Astr.Ap., submitted.

André P., Montmerle T., Stine P.C., Feigelson E.D., Klein K.L. 1988, Ap.J.335, 940. 
Beckwith S.V.W., Sargent A.I., Chini R.S., Güsten R. 1990, Astr.J. in press.

Bertout C. 1989, Ann.Rev.Astr.Ap., 27, 351.

Bertout C., Basri G., Bouvier J. 1988, Ap.J.330, 350.

Bieging J.H., Cohen M., Schwartz P.R. 1984, Ap.J.282, 699.

Bouvier J. 1987, Ph.D. Thesis, University Paris 7.

Bouvier J., Bertout C. 1989, Astr.Ap.211, 99.

Brown A. 1987, Ap.J.(Letters)322, L31.

Caillault J.P., Zoonematkermani S. 1987, in Circumstellar Matter, ed. I. Appenzeller and C. Jordan (Dordrecht: Reidel), p. 119.

Churchwell E., Felli M., Wood D.O.S., Massi M. 1987, Ap.J.321, 516.

Cohen M., Bieging J.H. 1986, Astr.J.92, 1396.

Edwards S., Cabrit S., Strom S.E., Heyer I., Strom K.M., Anderson E. 1987, Ap.J.321, 473.

Feigelson E.D., DeCampli W.M. 1981, Ap.J.(Letters)243, L89.

Feigelson E.D., Giampapa M.S., Vrba F.J. 1990, in The Sun in Time, eds. C.P. Sonett and M.S. Giampapa (Tucson: University of Arizona Press), in press.

Feigelson E.D., Kriss G.A. 1981, Ap.J.(Letters)248, L35.

Feigelson E.D., Jackson J.M., Mathieu R.D., Myers P.C., Walter F.M. 1988, Astr.J.94, 1251.

Feigelson E.D., Montmerle T. 1985, Ap.J.(Letters)289, L19.

Gahm G.F. 1990, This volume.

Garay G., Moran J.M., Reid M.J. 1987, Ap.J.314, 535.

Kenyon S.J., Hartmann L. 1987, Ap.J.323, 714.

Klein K.L., Chiuderi-Drago F. 1987, Astr.Ap.175, 179.

Ku W.H.-M., Righini-Cohen G., Simon M. 1982, Science215, 61.

Lada C.J. 1985, Ann.Rev.Astr.Ap. 23, 267.

Lada C.J. 1988, in Formation and Evolution of Low Mass Stars, ed. A.K. Dupree and M.T.V.T. Lago, NATO ASI Series (Dordrecht: Kluwer Academic Publishers), p.93.

Montmerle T. 1987, in Solar and Stellar Physics, Proc. 5th Eur. Solar Meeting, ed. E.H.

Schröter and M. Schüssler (Berlin: Springer), Lect. Notes in Phys.292, 117.

Montmerle T., André P. 1988, in Formation and Evolution of Low Mass Stars, ed. A.K. Dupree and M.T.V.T. Lago, NATO ASI Series (Dordrecht: Kluwer Academic Publishers), p. 225.

Montmerle T., André P. 1989, in Proc. ESO Workshop Low Mass Star Formation and Pre-Main Sequence Objects, ed. B. Reipurth (Garching: ESO), p. 407.

Montmerle T., Koch-Miramond L., Falgarone E., Grindlay J.E. 1983, Ap.J.269, 182.

Montmerle T., Koch-Miramond L., Falgarone E., Grindlay J.E. 1984, in Proc. Very Hot Astrophysical Plasmas, ed. L. Koch-Miramond and T. Montmerle, Phys. Scripta, T7, 59.

Mundt R. 1984, Ap.J. 280, 749.

Mutel R.L., Morris M.H., Doiron D.J., Lestrade J.-F. 1987, Astr.J.93, 1220.

Panagia N., Felli M. 1975, Astr.Ap. 39, 1.

Snell R.L., Bally J.B. 1986, Ap.J. 303, 683.

Stine P.C., Feigelson E.D., André P., Montmerle T. 1988, Astr.J. 96, 1394.

Tagger M., Henriksen R.N., Sygnet J.F., Pellat R. 1989, Ap.J.(Letters), in press.

Walter F.M. 1987, Pub.A.S.P.99, 31.

Walter F.M., Kuhi L.V. 1984, Ap.J.284, 194.

Walter F.M., Brown A., Mathieu R.D., Myers P.C., Vrba F.J. 1988, Astr.J.96, 297.

Wilking B.A., Lada C.J., Young E.T. 1989, Ap.J. 340, 823. 
LANG: Could the VLBI be resolving the H II region, and why would you expect thermal emission at radio wavelengths?

MONTMERLE: The H II region is much bigger (about 3"), and some thermal radio emission due to stellar winds have been detected from some $T$ Tauri stars.

BENZ: The interaction of an accretion disk and a magnetic field'would be a possible way to accelerate the radio emitting electrons (éxample: Jupiter's moon Io). Why don't you have an accretion disk in your model?

MONTMERLE: In the case of the Rho Oph radio sources, we have no observational indication of the presence of warm circumstellar material (i.e. near-IR excess). So we have proposed (see Andre et al., 1988, Ap. $\mathrm{J}, 335,940$ ) that these electrons could come from a weak ionized wind, combined with the star's rotation (see also the case of Jupiter's magnetosphere, rotating in the solar wind). This is very likely in the case of S1, which is a B star, more speculative in the case of DoAr 21, which is of a later type. But any weak wind of solar type (i.e. larger than $10 * *(-14)$ solarmasses per year) would presumably be sufficient.

PALLAVICINI: Knowing only the temperature and emission measure of the $\mathrm{X}$-ray source, how can you infer the size of the $\mathrm{x}$-ray emitting region?

MONTMERLE: In the particular case of the Rho Oph X-ray source ROX-20, this is possible because we know, in addition, the decay time of the flare (cooling by radiation); see Montmerle et al. 1983, Ap.J. 269, 182 .

PALLAVICINI: Could you explain the quiescent radio emission by thermal gyrosynchrotron by the same electrons responsible for the $\mathrm{X}$-ray corona?

MONTMERLE: This idea has recently been proposed by Linsky (see Drake et al., Ap.J. suppl., in press), on the basis of a correlation between the $6 \mathrm{~cm}$ radio flux and the $\mathrm{X}$-ray flux for $\mathrm{RS}$ CVn stars. However, recent work by Chiuderi-Drago and klein (11th IAU European Regional Astronomy Meeting, 1989) has shown that, unless very high surface magnetic fields (several kG at least) are present, it is not possible to account simultaneously for other radio data (for instance at $20 \mathrm{~cm}$ and $2 \mathrm{~cm}$ in the case of HR 1099 and UX Ari) when available. Such high magnetic fields are too large compared with the values of less than $1 \mathrm{kG}$ inferred from other works on these stars. So we stick to a nonthermal electron distribution.

GAHM: Did I understand correctly that you make a distinction between the nonthermal extended radio stars and the naked $\mathrm{T}$ Tauri stars?

MONTMERLE: Yes and no. In our Rho Oph sample we found radio emission only from stars embedded in the cloud core, which, presumably because of the heavy extinction, have little or no $\mathrm{X}$-ray emission. Conversely, down to a sensitivity of $2 \mathrm{mJy}$ ( 
we have not detected any of the Rox sources which are naked (or preferably "weak-line") T Tauri stars.

on the other hand, to this sensitivity in radio, only large emission measures (i.e. large sizes) are detectable. So it is likely that the present distinction only means that naked TTS have in general smaller magnetic loop sizes. Since we have noted (see Montmerle and Andre in "Formation and Evolution of Low-Mass Stars", NATO-ASI, Kluwer 1988, p. 225) that nonthermal radio emitters have high rotation velocities (more than $80 \mathrm{~km} / \mathrm{s}$, although measured on an admittedly small sample) it is quite possible that the bulk of the naked TTS have smaller loop sizes because they are slow rotators. However, a deeper survey of radio emission from NTTS is still missing.

TSVETKOV: Is the object DOAr 21 a T Tauri star?

MONTMERLE: Strictly speaking, no, since it appears to be more massive (about 2 solar masses). But it seems to be sufficiently high above the main sequence that it is still on a convective track, hence its basic properties should not differ drastically from those of standard $T$ Tauri stars (about 1 solar mass or less). 